\title{
Bounds for Generalized Distance Spectral Radius and the Entries of the Principal Eigenvector
}

\author{
Abdollah Alhevaz, Maryam Baghipur, Hilal Ahmad Ganie and Gui-Xian Tian
}

\begin{abstract}
For a simple connected graph $G$, the convex linear combinations $D_{\alpha}(G)$ of $\operatorname{Tr}(G)$ and $D(G)$ is defined as $D_{\alpha}(G)=\alpha \operatorname{Tr}(G)+(1-\alpha) D(G), 0 \leq \alpha \leq 1$. As $D_{0}(G)=D(G)$, $2 D_{\frac{1}{2}}(G)=D^{Q}(G), D_{1}(G)=\operatorname{Tr}(G)$ and $D_{\alpha}(G)-D_{\beta}(G)=(\alpha-\beta) D^{L}(G)$, this matrix reduces to merging the distance spectral and distance signless Laplacian spectral theories. In this paper, we study the spectral properties of the generalized distance matrix $D_{\alpha}(G)$. We obtain some lower and upper bounds for the generalized distance spectral radius, involving different graph parameters and characterize the extremal graphs. Further, we obtain upper and lower bounds for the maximal and minimal entries of the $p$-norm normalized Perron vector corresponding to spectral radius $\partial(G)$ of the generalized distance matrix $D_{\alpha}(G)$ and characterize the extremal graphs.
\end{abstract}

\section{Introduction}

In this paper, we consider only connected, undirected, simple and finite graphs. A graph is denoted by $G=(V(G), E(G))$, where $V(G)=\left\{v_{1}, v_{2}, \ldots, v_{n}\right\}$ is its vertex set and $E(G)$ is its edge set. The order of $G$ is the number $n=|V(G)|$ and its size is the number $m=|E(G)|$. The set of vertices adjacent to $v \in V(G)$, denoted by $N(v)$, refers to the neighborhood of $v$. The degree of $v$, denoted by $d_{G}(v)$ (we simply write $d_{v}$ if it is clear from the context) means the cardinality of $N(v)$. A graph is called regular if each of its vertex has the same degree. The distance between two vertices $u, v \in V(G)$, denoted by $d_{u v}$, is defined as the length of a shortest path between $u$ and $v$ in $G$. The diameter of $G$ is the maximum distance between any two vertices of $G$. The distance matrix of $G$ is denoted by $D(G)$ and is defined as $D(G)=\left(d_{u v}\right)_{u, v \in V(G)}$. The transmission $\operatorname{Tr}_{G}(v)$ of a vertex $v$ is defined to be the sum of the distances from $v$ to all other vertices in $G$, i.e., $\operatorname{Tr}_{G}(v)=\sum_{u \in V(G)} d_{u v}$. A graph $G$ is said to be $k$-transmission regular if $\operatorname{Tr}_{G}(v)=k$, for each $v \in V(G)$. The transmission of a graph $G$, denoted by $W(G)$, is the sum of distances

Key words and phrases. Distance matrix (spectrum); Distance signlees Laplacian matrix (spectrum); generalized distance matrix; spectral radius; transmission regular graph; Perron vector.

2010 Mathematics Subject Classification. Primary : 05C50, 05C12; Secondary: 15A18.

Corresponding author: Hilal Ahmad Ganie. 
between all unordered pairs of vertices in $G$. Clearly, $W(G)=\frac{1}{2} \sum_{v \in V(G)} \operatorname{Tr}_{G}(v)$. For any vertex $v_{i} \in V(G)$, the transmission $\operatorname{Tr}_{G}\left(v_{i}\right)$ is called the transmission degree, shortly denoted by $\operatorname{Tr}_{i}$ and the sequence $\left\{T r_{1}, T r_{2}, \ldots, T r_{n}\right\}$ is called the transmission degree sequence of the graph $G$. The second transmission degree of $v_{i}$, denoted by $T_{i}$ is given by $T_{i}=\sum_{j=1}^{n} d_{i j} T r_{j}$.

Let $\operatorname{Tr}(G)=\operatorname{diag}\left(\operatorname{Tr}_{1}, \operatorname{Tr}_{2}, \ldots, T r_{n}\right)$ be the diagonal matrix of vertex transmissions of $G$. M. Aouchiche and P. Hansen [1, 2] introduced the Laplacian and the signless Laplacian for the distance matrix of a connected graph. The matrix $D^{L}(G)=\operatorname{Tr}(G)-D(G)$ is called the distance Laplacian matrix of $G$, while the matrix $D^{Q}(G)=\operatorname{Tr}(G)+D(G)$ is called the distance signless Laplacian matrix of $G$. The spectral properties of $D(G), D^{L}(G)$ and $D^{Q}(G)$ have attracted much more attention of the researchers and a large number of papers have been published regarding their spectral properties, like spectral radius, second largest eigenvalue, smallest eigenvalue etc. For some recent works we refer to $[3,8,4,15,17,18,19,20,21,22,25,26,27]$ and the references therein.

Recently in [9], Cui et al. introduced the generalized distance matrix $D_{\alpha}(G)$ as a convex combinations of $\operatorname{Tr}(G)$ and $D(G)$, defined as $D_{\alpha}(G)=\alpha \operatorname{Tr}(G)+(1-\alpha) D(G)$, for $0 \leq \alpha \leq 1$. Since $D_{0}(G)=D(G), \quad 2 D_{\frac{1}{2}}(G)=D^{Q}(G), \quad D_{1}(G)=\operatorname{Tr}(G)$ and $D_{\alpha}(G)-D_{\beta}(G)=$ $(\alpha-\beta) D^{L}(G)$, any result regarding the spectral properties of generalized distance matrix, has its counterpart for each of these particular graph matrices, and these counterparts follow immediately from a single proof. In fact, this matrix reduces to merging the distance spectral and distance signless Laplacian spectral theories. Since the matrix $D_{\alpha}(G)$ is real symmetric, all its eigenvalues are real. Therefore, we can arrange them as $\partial_{1} \geq \partial_{2} \geq \cdots \geq \partial_{n}$. The largest eigenvalue $\partial_{1}$ of the matrix $D_{\alpha}(G)$ is called the generalized distance spectral radius of $G$ (From now onwards, we will denote $\partial_{1}(G)$ by $\left.\partial(G)\right)$. As $D_{\alpha}(G)$ is nonnegative and irreducible, by the Perron-Frobenius theorem, $\partial(G)$ is the unique eigenvalue and there is a unique positive unit eigenvector $X$ corresponding to $\partial(G)$, which is called the generalized distance Perron vector of $G$. For some recent papers on the generalized distance eigenvalues of a graph, we refer to $[5,6,7,16]$ and the references therein.

The spectral radius of a general matrix $M$ is a very attractive and interesting topic of research and as such the investigation of the spectral radius of some matrices associated with a graph become interesting. When $M$ is restricted to a particular graph matrix, the spectral radius has attracted much attention of the researchers as is clear from the fact that various papers can be found in the literature in this direction. For a particular graph matrix (like adjacency, Laplacian, signless Laplacian, etc), the much studied problem about the parameter spectral radius is to obtain bounds in terms of various graph parameters. Another problem worth to mention is to characterize the extremal graphs for the spectral radius of a graph matrix, in some classes of graphs. For some recent works we refer to $[15,19,20,22,25,26,27]$ and the references therein. 
A column vector $X=\left(x_{1}, x_{2}, \ldots, x_{n}\right)^{T} \in \mathbb{R}^{n}$ can be considered as a function defined on $V(G)$ which maps vertex $v_{i}$ to $x_{i}$, i.e., $X\left(v_{i}\right)=x_{i}$ for $i=1,2, \ldots, n$. Then,

$$
X^{T} D_{\alpha}(G) X=\alpha \sum_{i=1}^{n} \operatorname{Tr}\left(v_{i}\right) x_{i}^{2}+2(1-\alpha) \sum_{1 \leq i<j \leq n} d\left(v_{i}, v_{j}\right) x_{i} x_{j},
$$

and $\lambda$ is an eigenvalue of $D_{\alpha}(G)$ corresponding to the eigenvector $X$ if and only if $X \neq \mathbf{0}$ and,

$$
\lambda x_{i}=\alpha \operatorname{Tr}\left(v_{i}\right) x_{i}+(1-\alpha) \sum_{j=1}^{n} d\left(v_{i}, v_{j}\right) x_{j}
$$

These equations are called the $(\lambda, x)$-eigenequations of $G$. For a normalized column vector $X \in$ $\mathbb{R}^{n}$ with at least one non-negative component, by the Rayleigh's principle, we have

$$
\partial(G) \geq X^{T} D_{\alpha}(G) X
$$

with equality if and only if $X$ is the generalized distance Perron vector of $G$.

One of the interesting problems that can be found in the literature is regarding the bounds for the minimal and maximal entry of the Perron vector of the graph $G$. The problem is that, for a particular graph matrix find upper and lower bounds for the minimal and maximal entry of the Perron vector of the graph $G$ and characterize the extremal graphs. The principal eigenvector is of interest since it is often used in applications, see [24] for an overview. For a fixed real number $p, 1 \leq p<\infty$, the unique positive eigenvector $X=\left(x_{1}, x_{2}, \ldots, x_{n}\right)^{T}$ such that $\left(\sum_{i=1}^{n} x_{i}^{p}\right)^{\frac{1}{p}}=$ 1 associated with the largest eigenvalue of a symmetric, non-negative and irreducible matrix, is called the $p$-normalized principal eigenvector of the matrix. Let us denote the maximum and minimum entry of $X$ of by $x_{\max }$ and $x_{\min }$, respectively. Several results regarding the maximal and minimal entries of the principal eigenvector are available in the literature, here we mention some of them. Papendieck and Recht [23] obtained an upper bound on the maximal entry of the principal eigenvector of adjacency matrix of a simple connected graph and the bound depends only on the adjacency spectral radius of the graph. Zhao and Hong [28] investigated the maximal entry of the $p$-normalized principal eigenvector of symmetric non-negative matrix with zero trace and gave both upper and lower bounds for the same. In [11], Cioabă and Gregory found an upper bound of the maximal entry of the principal eigenvector of adjacency matrix in terms of the maximum degree of the graph and showed that their upper bound improves the result of Papendieck and Recht [23]. In [10], Cioabă gave a necessary and sufficient condition for a graph to be bipartite in terms of principal eigenvector of the adjacency matrix of the graph. Das [12] obtained a sharp upper bound on the maximum entry of the $p$-normalized principal eigenvector for a symmetric non-negative matrix in terms of the order, spectral radius, largest and smallest diagonal entries of the matrix. Moreover, in the same paper [12], Das found an upper bound on 
the maximum entry of the $p$-normalized principal eigenvector for the signless Laplacian matrix of a graph. Further in [13] Das determined lower and upper bounds on the maximum entry and an upper bound on minimum entry of the $p$-normalized principal eigenvector for the distance matrix of a graph and characterized the extremal graphs. In 2015 Das et al. [14] considered the distance signless Laplacian and determined upper and lower bounds on the maximum entry and an upper bound on minimum entry of the $p$-normalized principal eigenvector and characterized the extremal graphs. Recently, Atik and Panigrahi [8] obtained some upper and lower bounds of the maximal and minimal entry of the $p$-normalized principal eigenvector for the distance matrix and distance signless Laplacian matrix of a graph and showed that transmission regular graphs are extremal for all these bounds. They also show that their bounds improve the bounds obtained in [13] and [14] for some families of graphs.

Motivated by the works mentioned above, in this paper we study the spectral radius of generalized distance matrix $D_{\alpha}(G)$ and obtain some upper and lower bounds in terms of various graph parameters. We also study the entries of the $p$-normalized principal eigenvector of the generalized distance matrix $D_{\alpha}(G)$ and obtain some lower and upper bounds in terms of several graph parameters. Moreover, we characterize the extremal graphs attaining these bounds. Since $D_{0}(G)=D(G)$ and $2 D_{\frac{1}{2}}(G)=D^{Q}(G)$, therefore most of our results generalize the results obtained in $[8,13,14]$.

The rest of the paper is organized as follows. In Section 2, we obtain some bounds for the generalized distance spectral radius of graphs and characterize the extremal graphs. In Section 3, we obtain upper and lower bounds for the minimal and maximal entry of the generalized distance Perron vector of the graph $G$ and characterize the extremal graphs. Our results in this section generalizes many of the existing results in the literature, especially the results in the references $[8,13,14]$ to a more general setting.

\section{Bounds on generalized distance spectral radius}

In this section, we obtain upper and lower bounds for the generalized spectral radius $\partial(G)$, in terms of the transmission degree sequence and the second transmission degree sequence, the diameter and the order of the graph $G$.

Theorem 2.1. Let $X=\left(x_{1}, x_{2}, \ldots, x_{n}\right)^{T}$ be the generalized distance Perron vector of graph $G$. If $x_{i}=\max \left\{x_{k} \mid k=1,2, \ldots, n\right\}$ and $x_{j}=\min \left\{x_{k} \mid k=1,2, \ldots, n\right\}$, then

$$
\operatorname{Tr}_{j} \leq \partial(G) \leq T r_{i}
$$

Equality occurs on both sides if and only if $G$ is a transmission regular graph. 
Proof. From the $i$-th eigenequation we have,

$$
\begin{gathered}
\partial(G) x_{i}=\alpha T r_{i} x_{i}+(1-\alpha) \sum_{j=1}^{n} d_{i j} x_{j} \\
\Rightarrow \partial(G)-\alpha T r_{i}=(1-\alpha) \sum_{j=1}^{n} d_{i j} \frac{x_{j}}{x_{i}} \\
\Rightarrow \partial(G)-\alpha T r_{i} \leq(1-\alpha) T r_{i} \text { and } \partial(G)-\alpha T r_{i} \geq(1-\alpha) T r_{j} \\
\Rightarrow \operatorname{Tr}_{j} \leq \partial(G) \leq T r_{i} .
\end{gathered}
$$

Suppose that equality occurs on the right side of (2.1), then $X$ is an eigenvector of $\partial(G)$ with $x_{i}=x_{k}$ for all $k=1,2, \ldots, n$. This gives all the row sums of $D_{\alpha}(G)$ are same, and so $G$ is a transmission regular graph. The equality on the left side can be discussed similarly.

Since for a graph with diameter at most two, we have $\operatorname{Tr}_{i}=2 n-2-d_{i}$, for all $i=1,2, \ldots, n$, it follows that a graph $G$ with diameter at most two is transmission regular if and only if it is degree regular. The following observation gives a lower bound for $\partial(G)$, in terms of the order $n$ and the maximum degree $\Delta$, and follows from Theorem 2.1.

Corollary 2.2. If $\Delta$ denotes the maximum degree of a graph $G$, then

$$
\partial(G) \geq 2 n-\Delta-2,
$$

with equality if and only if $G$ is a regular graph with diameter at most 2.

Proof. Using Theorem 2.1 and the fact that $T r_{i} \geq d_{i}+2\left(n-d_{i}-1\right)=2 n-d_{i}-2$, with equality if and only if $G$ is a regular graph with diameter at most 2, the result follows.

The following gives a lower bound for $\partial(G)$, in terms of the order $n$, the maximum degree $\Delta_{1}$ and the second maximum degree $\Delta_{2}$ of the graph $G$.

Theorem 2.3. Let $G$ be a connected graph of order $n$ having maximum degree $\Delta_{1}$ and second maximum degree $\Delta_{2}$. Then for $s=\Delta_{1}+\Delta_{2}$

$$
\partial(G) \geq \frac{\alpha(4 n-4-s)+\sqrt{\alpha^{2}(4 n-4-s)^{2}+4(1-2 \alpha)\left(2 n-2-\Delta_{1}\right)\left(2 n-2-\Delta_{2}\right)}}{2},
$$

with equality if and only if $G$ is a regular graph with diameter at most two.

Proof. Let $X=\left(x_{1}, x_{2}, \ldots, x_{n}\right)^{T}$ be the generalized distance Perron vector of graph $G$ and let $x_{i}=\min \left\{x_{k} \mid k=1,2, \ldots, n\right\}$ and $x_{j}=\min _{k \neq i}\left\{x_{k} \mid k=1,2, \ldots, n\right\}$. From the $i$-th equation of $D_{\alpha}(G) X=\partial(G) X$, we obtain

$$
\partial x_{i}=\alpha \operatorname{Tr}_{i} x_{i}+(1-\alpha) \sum_{k=1, k \neq i}^{n} d_{i k} x_{k} \geq \alpha \operatorname{Tr}_{i} x_{i}+(1-\alpha) \operatorname{Tr}_{i} x_{j}
$$


Similarly, from the $j$-th equation of $D_{\alpha}(G) X=\partial(G) X$, we obtain

$$
\partial x_{j}=\alpha \operatorname{Tr}_{j} x_{j}+(1-\alpha) \sum_{k=1, k \neq j}^{n} d_{j k} x_{k} \geq \alpha \operatorname{Tr}_{j} x_{j}+(1-\alpha) \operatorname{Tr}_{j} x_{i}
$$

Since

$$
\operatorname{Tr}_{p}=\sum_{p=1, k \neq p}^{n} d_{j p} \geq d_{p}+2\left(n-1-d_{p}\right)=2 n-2-d_{p}, \quad \text { for } \text { all } p=1,2, \ldots, n
$$

it follows that

$$
\left(\partial-\alpha\left(2 n-2-d_{i}\right)\right) x_{i} \geq(1-\alpha)\left(2 n-2-d_{i}\right) x_{j}
$$

and

$$
\left(\partial-\alpha\left(2 n-2-d_{j}\right)\right) x_{j} \geq(1-\alpha)\left(2 n-2-d_{j}\right) x_{i}
$$

Multiplying the corresponding sides of these inequalities and using the fact that $x_{k}>0$ for all $k$, we obtain

$$
\partial^{2}-\alpha\left(4 n-4-d_{i}-d_{j}\right) \partial-(1-2 \alpha)\left(2 n-2-d_{i}\right)\left(2 n-2-d_{j}\right) \geq 0
$$

which in turn gives

$\partial(G) \geq \frac{\alpha\left(4 n-4-d_{i}-d_{j}\right)+\sqrt{\alpha^{2}\left(4 n-4-d_{i}-d_{j}\right)^{2}+4(1-2 \alpha)\left(2 n-2-d_{i}\right)\left(2 n-2-d_{j}\right)}}{2}$.

Now, using $d_{i}+d_{j} \leq \Delta_{1}+\Delta_{2}$, the result follows.

Suppose that equality occurs in (2.2), then equality occurs in each of the above inequalities. If equality occurs in (2.3) and (2.4), then we obtain $x_{i}=x_{k}$, for all $k=1,2, \ldots, n$ giving that $G$ is a transmission regular graph. Also equality in (2.5) gives that $G$ is a graph of diameter at most two and equality in $d_{i}+d_{j} \leq \Delta_{1}+\Delta_{2}$ gives that $G$ is a regular graph. Combining all these, it follows that equality occurs in (2.2) if $G$ is a regular graph of diameter at most two.

Conversely, if $G$ is a connected $\Delta$-regular graph of diameter at most two, then $\partial(G)=T r_{i}=$ $2 n-2-\Delta$. Also

$$
\begin{aligned}
& \frac{\alpha(4 n-4-s)+\sqrt{\alpha^{2}(4 n-4-s)^{2}+4(1-2 \alpha)\left(2 n-2-\Delta_{1}\right)\left(2 n-2-\Delta_{2}\right)}}{2} \\
& =\frac{\alpha(4 n-4-s)+\alpha(4 n-4-s)(1-\alpha)}{2}=2 n-2-\Delta=\partial(G) .
\end{aligned}
$$

That completes the proof. 
Remark 1. For any connected graph $G$ of order $n$ having maximum degree $\Delta$, the lower bound given by Theorem 2.3 is always better than the lower bound given by Corollary 2.2. As

$$
\begin{array}{r}
\frac{\alpha(4 n-4-s)+\sqrt{\alpha^{2}(4 n-4-s)^{2}+4(1-2 \alpha)\left(2 n-2-\Delta_{1}\right)\left(2 n-2-\Delta_{2}\right)}}{2} \\
\geq \frac{\alpha\left(4 n-4-2 \Delta_{1}\right)+\sqrt{\alpha^{2}\left(4 n-4-2 \Delta_{1}\right)^{2}+4(1-2 \alpha)\left(2 n-2-\Delta_{1}\right)^{2}}}{2} \\
=\frac{\alpha\left(4 n-4-2 \Delta_{1}\right)+\alpha(4 n-4-s)(1-\alpha)}{2}=2 n-2-\Delta .
\end{array}
$$

The following gives an upper bound for $\partial(G)$, in terms of transmission degrees $\operatorname{Tr}_{i}$ of the graph $G$.

Theorem 2.4. Let $G$ be connected graph of order $n$ having transmission degree sequence $\left\{\operatorname{Tr}_{1}, \operatorname{Tr}_{2}, \ldots, \operatorname{Tr}_{n}\right\}$. Then

$$
\partial(G) \leq \max _{1 \leq i \neq j \leq n}\left\{\alpha T r_{i}+(1-\alpha) T r_{j}\right\}
$$

with equality if and only if $G$ is a transmission regular graph.

Proof. Let $X=\left(x_{1}, x_{2}, \ldots, x_{n}\right)^{T}$ be an eigenvector of $\operatorname{Tr}(G)^{-1} D_{\alpha}(G) \operatorname{Tr}(G)$ corresponding to $\partial(G)$ and $x_{i}=\max \left\{x_{j} \mid j=1,2, \ldots, n\right\}$. The $(i, j)$-th entry of $\operatorname{Tr}(G)^{-1} D_{\alpha}(G) \operatorname{Tr}(G)$ is

$$
\left\{\begin{aligned}
\alpha T r_{i} & \text { if } i=j \\
\frac{T r_{j}}{T r_{i}}(1-\alpha) d_{i j} & \text { otherwise. }
\end{aligned}\right.
$$

We have

$$
\operatorname{Tr}(G)^{-1} D_{\alpha}(G) \operatorname{Tr}(G) X=\partial(G) X
$$

From the $i$-th equation of (2.7), we have

$$
\begin{aligned}
\partial(G) x_{i} & =\alpha T r_{i} x_{i}+(1-\alpha) \sum_{j=1, j \neq i}^{n} \frac{T r_{j} d_{i j}}{T r_{i}} x_{j} \\
\Rightarrow\left(\partial(G)-\alpha T r_{i}\right) x_{i} & =(1-\alpha) \sum_{j=1, j \neq i}^{n} \frac{T r_{j} d_{i j}}{T r_{i}} x_{j} \\
\Rightarrow x_{i}\left(\partial(G)-\alpha T r_{i}\right) x_{i} & =(1-\alpha) \sum_{j=1, j \neq i}^{n} \frac{T r_{j}}{T r_{i}} x_{i} d_{i j} x_{j} \leq(1-\alpha) x_{i}^{2} \sum_{j=1}^{n} \frac{T r_{j}}{T r_{i}} d_{i j} \\
\Rightarrow \partial(G) & \leq \alpha T r_{i}+\frac{1-\alpha}{T r_{i}} \sum_{j=1, j \neq i}^{n} \operatorname{Tr}_{j} d_{i j}
\end{aligned}
$$




$$
\begin{aligned}
& \leq \alpha \operatorname{Tr}_{i}+\frac{1-\alpha}{\operatorname{Tr}_{i}} \max _{1 \leq j \neq i \leq n}\left\{T r_{j}\right\} \sum_{j=1}^{n} d_{i j} \\
& \leq \alpha T r_{i}+(1-\alpha) \max _{1 \leq j \leq n}\left\{\operatorname{Tr}_{j}\right\} \\
& \leq \max _{1 \leq i, j \leq n}\left\{\alpha T r_{i}+(1-\alpha) \operatorname{Tr}_{j}\right\} .
\end{aligned}
$$

Which completes the proof of inequality (2.6). Now, suppose that equality in (2.6) holds, then all inequalities in the above argument must be equalities. From equality in (2.8), we get $x_{1}=$ $x_{2}=\cdots=x_{n}$, giving that $G$ is a transmission regular graph. From equality in (2.9), we get $T r_{1}=T r_{2}=\cdots=T r_{n}=\max _{1 \leq j \neq i \leq n}\left\{T r_{j}\right\}$. Let $T r_{s}:=\max _{1 \leq j \neq i \leq n}\left\{\operatorname{Tr}_{j}\right\}$, then either $\operatorname{Tr}_{i}=\operatorname{Tr}_{s}$ or $T r_{i} \neq T r_{s}$. If $T r_{i}=T r_{s}$, then all the transmissions of the vertices are equal and $G$ is a transmission regular graph. On the other hand, if $T r_{i} \neq T r_{s}$, then the graph $G$ is not a transmission regular graph and so equality can not occur in this case.

Conversely, suppose that $G$ is a transmission regular graph, then it can easily seen that equality occurs in (2.6). That completes the proof.

The following gives another upper bound for $\partial(G)$, in terms of transmission degree sequence and the second transmission degree sequence of the graph $G$.

Theorem 2.5. If the transmission degree sequence and the second transmission degree sequence of $G$ are $\left\{\operatorname{Tr}_{1}, \operatorname{Tr}_{2}, \ldots, \operatorname{Tr}_{n}\right\}$ and $\left\{T_{1}, T_{2}, \ldots, T_{n}\right\}$, respectively, then

$$
\partial(G) \leq \max _{v_{i} \in V(G)}\left\{\frac{\alpha T r_{i}+\sqrt{\Theta}}{2}\right\}
$$

where $\Theta=\alpha^{2} \operatorname{Tr}_{i}^{2}+\frac{4(1-\alpha)}{T r_{i}} \sum_{j=1}^{n} d_{i j}\left((1-\alpha) T_{j}+\alpha T r_{j}^{2}\right)$. Moreover, if $\frac{1}{2} \leq \alpha<1$, the equality holds if and only if $G$ is a transmission regular graph.

Proof. Let $X=\left(x_{1}, x_{2}, \ldots, x_{n}\right)^{T}$ be an eigenvector corresponding to the eigenvalue $\partial(G)$ of $\operatorname{Tr}^{-1}(G) D_{\alpha}(G) \operatorname{Tr}(G)$. We assume that one eigencomponent $x_{i}$ is equal to 1 and the other eigencomponents are less than or equal to 1 , that is $x_{k}<1$, for all $k=1,2, \ldots, n, k \neq i$. The $(i, j)$-th entry of $\operatorname{Tr}(G)^{-1} D_{\alpha}(G) \operatorname{Tr}(G)$ is

$$
\left\{\begin{aligned}
\alpha T r_{i} & \text { if } i=j \\
\frac{T r_{j}}{T r_{i}}(1-\alpha) d_{i j} & \text { otherwise. }
\end{aligned}\right.
$$

We have

$$
\operatorname{Tr}(G)^{-1} D_{\alpha}(G) \operatorname{Tr}(G) X=\partial(G) X
$$


From the $i$-th equation of (2.11), we have

$$
\begin{aligned}
& \partial(G) x_{i}=\alpha T r_{i} x_{i}+\sum_{j=1}^{n} \frac{T r_{j}}{T r_{i}}(1-\alpha) d_{i j} x_{j}, \\
& \Rightarrow \partial(G)=\alpha T r_{i}+\sum_{j=1}^{n} \frac{T r_{j}}{T r_{i}}(1-\alpha) d_{i j} x_{j} .
\end{aligned}
$$

Similarly from the $j$-th equation of (2.11), we have

$$
\partial(G) x_{j}=\alpha \operatorname{Tr}_{j} x_{j}+\sum_{k=1}^{n} \frac{\operatorname{Tr}_{k}}{\operatorname{Tr}_{j}}(1-\alpha) d_{j k} x_{k} .
$$

Multiplying both sides of (2.12) by $\partial(G)$ and substituting the value of $\partial(G) x_{j}$, we get

$$
\begin{aligned}
\partial^{2}(G) & =\alpha \operatorname{Tr}_{i} \partial(G)+\sum_{j=1}^{n}\left\{\frac{\operatorname{Tr}_{j}}{\operatorname{Tr}_{i}}(1-\alpha) d_{i j}\left[\alpha T r_{j} x_{j}+\sum_{k=1}^{n} \frac{\operatorname{Tr}_{k}}{\operatorname{Tr}_{j}}(1-\alpha) d_{j k} x_{k}\right]\right\} \\
& =\alpha \operatorname{Tr}_{i} \partial(G)+\alpha(1-\alpha) \sum_{j=1}^{n} \frac{\operatorname{Tr}_{j}^{2}}{T r_{i}} d_{i j} x_{j}+(1-\alpha)^{2} \sum_{j=1}^{n} \sum_{k=1}^{n} \frac{\operatorname{Tr}_{k}}{\operatorname{Tr}_{i}} d_{i j} d_{j k} x_{k} \\
& \leq \alpha \operatorname{Tr}_{i} \partial(G)+\alpha(1-\alpha) \sum_{j=1}^{n} \frac{\operatorname{Tr}_{j}^{2}}{\operatorname{Tr}_{i}} d_{i j}+(1-\alpha)^{2} \sum_{j=1}^{n} \frac{T_{j}}{\operatorname{Tr}_{i}} d_{i j} \\
& =\alpha \operatorname{Tr}_{i} \partial(G)+\frac{1-\alpha}{\operatorname{Tr}_{i}} \sum_{j=1}^{n} d_{i j}\left((1-\alpha) T_{j}+\alpha \operatorname{Tr}_{j}^{2}\right) .
\end{aligned}
$$

This shows that

$$
\partial^{2}(G)-\alpha \operatorname{Tr}_{i} \partial(G)-\frac{1-\alpha}{\operatorname{Tr}_{i}} \sum_{j=1}^{n} d_{i j}\left((1-\alpha) T_{j}+\alpha \operatorname{Tr}_{j}^{2}\right) \leq 0
$$

From this the result now follows. Suppose that the equality holds in (2.10). Then all inequalities in the above argument must be equalities. From equality in (2.13), we get $x_{j}=1$ for all $j$. From this one can easily show that $x_{i}=1$ for all $i \in V$. Hence

$$
\alpha \operatorname{Tr}_{1}+\frac{(1-\alpha) T_{1}}{T r_{1}}=\alpha \operatorname{Tr}_{2}+\frac{(1-\alpha) T_{2}}{T r_{2}}=\cdots=\alpha T r_{n}+\frac{(1-\alpha) T_{n}}{T r_{n}} .
$$

Let $T r_{\max }$ and $T r_{\min }$ denote the maximum and minimum vertex transmission, respectively. Without loss of generality, assume that $T r_{i}=T r_{\max }$ and $T r_{j}=T r_{\min }$. Therefore,

$$
\alpha T r_{\max }+\frac{(1-\alpha) T_{i}}{T r_{\max }}=\alpha T r_{\min }+\frac{(1-\alpha) T_{j}}{T r_{\min }}
$$

Since $T_{i} \geq T r_{\max } T r_{\min }$ and $T_{j} \leq T r_{\max } T r_{\min }$, it follows that

$\alpha T r_{\max }+(1-\alpha) T r_{\min } \leq \alpha T r_{\max }+\frac{(1-\alpha) T_{i}}{T r_{\max }}=\alpha T r_{\min }+\frac{(1-\alpha) T_{j}}{T r_{\min }} \leq(1-\alpha) T r_{\max }+\alpha T r_{\min }$.

This gives that $T r_{\max }=T r_{\min }$ for $\frac{1}{2} \leq \alpha<1$. Hence $G$ is a transmission regular graph.

Conversely, suppose that for $\frac{1}{2} \leq \alpha<1$ the graph $G$ is a transmission regular graph, then it can easily seen that equality occurs in (2.10). This completes the proof. 


\section{Bounds for the entries in the Perron vector of the generalized distance matrix}

In this section, we obtain upper and lower bounds on the minimal and maximal entries of the $p$-norm normalized Perron vector corresponding to spectral radius $\partial(G)$ and characterize the extremal graphs.

The $p$-norm of a vector $X=\left(x_{1}, x_{2}, \ldots, x_{n}\right)^{T}$ is defined as follows:

$$
\|X\|_{p}=\left(\left|x_{1}\right|^{p}+\left|x_{2}\right|^{p}+\cdots+\left|x_{n}\right|^{p}\right)^{\frac{1}{p}}, 1 \leq p<\infty .
$$

For a fixed real number $p, 1 \leq p<\infty$, the unique positive eigenvector $X=\left(x_{1}, x_{2}, \ldots, x_{n}\right)^{T}$ such that $\left(\sum_{i=1}^{n} x_{i}^{p}\right)^{\frac{1}{p}}=1$ associated with the largest eigenvalue of a symmetric, non-negative and irreducible matrix, is called the $p$-normalized principal eigenvector of the matrix.

The following gives a lower bound and an upper bound for the minimal entry $x_{\min }$ of the Perron vector for $\partial(G)$.

Theorem 3.1. Let $G$ be a connected graph of order $n$ and let $X=\left(x_{1}, x_{2}, \ldots, x_{n}\right)^{T}$ be the Perron vector corresponding to spectral radius $\partial(G)$ of $D_{\alpha}(G)$ with $x_{1} \geq x_{2} \geq \ldots \geq x_{n}$. Then for $0 \leq \alpha<1$,

$$
\frac{1-\alpha}{\partial-T r_{\min }+n(1-\alpha)} \leq x_{n}=x_{\min } \leq \frac{\partial-\alpha T r_{\min }}{(1-\alpha) 2 W}
$$

Equality occurs for both the inequalities if and only if $G$ is a transmission regular graph.

Proof. By given $X=\left(x_{1}, x_{2}, \ldots, x_{n}\right)^{T}$ is the unit eigenvector corresponding to the eigenvalue $\partial(G)$ of the matrix $D_{\alpha}$, therefore

$$
D_{\alpha} X=\partial X, \quad \sum_{k=1}^{n} x_{k}=1
$$

giving that

$$
\begin{gathered}
\partial(G) x_{i}=\alpha T r_{i} x_{i}+(1-\alpha) \sum_{k=1, k \neq i}^{n} d_{i k} x_{k} \\
\Rightarrow\left(\partial(G)-\alpha T r_{\min }\right) x_{i} \geq(1-\alpha) \sum_{k=1, k \neq i}^{n} d_{i k} x_{k} .
\end{gathered}
$$

Summing over all $i$ and using the fact $\sum_{k=1}^{n} x_{k}=1$, we get

$$
\left(\partial(G)-\alpha T r_{\min }\right) \geq(1-\alpha) \sum_{i=1}^{n} \sum_{k=1, k \neq i}^{n} d_{i k} x_{k}
$$




$$
\geq(1-\alpha) 2 W x_{\min }
$$

From this the right hand inequality follows.

Again from (3.2), we have

$$
\begin{gathered}
\left(\partial(G)-\alpha T r_{\min }+(1-\alpha)\right) x_{i} \geq(1-\alpha) x_{i}+(1-\alpha) \sum_{k=1, k \neq i}^{n} d_{i k} x_{k} \\
\Rightarrow\left(\partial(G)-\alpha T r_{\min }+(1-\alpha)\right) x_{i} \geq(1-\alpha) \sum_{k=1}^{n} x_{k}+(1-\alpha) \sum_{k=1, k \neq i}^{n}\left(d_{i k}-1\right) x_{k} .
\end{gathered}
$$

Since this inequality is true for all $i$, therefore we have

$$
\left(\partial(G)-\alpha T r_{\min }+(1-\alpha)\right) x_{\min } \geq(1-\alpha)+(1-\alpha)\left(T r_{\min }-(n-1)\right) x_{\min }
$$

From this the left hand inequality follows.

Suppose that equality occurs in the left hand inequality of (3.1), then equality occurs in (3.2), giving that $T r_{i}=T r_{\min }$ and $x_{\min }=x_{i}$, for all $i=1,2, \ldots, n$. This shows that all the row sums of the matrix $D_{\alpha}(G)$ are equal, implying that $G$ is a transmission regular graph. Similarly, it can be seen that equality occurs in the left hand inequality of (3.1), if $G$ is a transmission regular graph.

Conversely, if $G$ is a transmission regular graph, then $T r_{\min }=\partial, 2 W=n T r_{\min }$ and $X=$ $\left(\frac{1}{n}, \frac{1}{n}, \ldots, \frac{1}{n}\right)^{T}$ is the unit vector corresponding to eigenvalue $\partial$ of the matrix $D_{\alpha}(G)$. We have

$$
\frac{1-\alpha}{\partial-T r_{\min }+n(1-\alpha)}=\frac{1}{n}=\frac{\partial-\alpha T r_{\min }}{(1-\alpha) 2 W}
$$

That completes the proof.

The following gives a lower and an upper bound for the maximal entry $x_{\max }$ of the Perron vector for $\partial(G)$.

Theorem 3.2. Let $G$ be a connected graph of order $n$ and let $X=\left(x_{1}, x_{2}, \ldots, x_{n}\right)^{T}$ be the Perron vector corresponding to spectral radius $\partial(G)$ of $D_{\alpha}(G)$ with $x_{1} \geq x_{2} \geq \ldots \geq x_{n}$. Then for $0 \leq \alpha<1$,

$$
\frac{\partial-\alpha T r_{\max }}{2 W(1-\alpha)} \leq x_{1}=x_{\max } \leq \frac{(1-\alpha) T r_{\max }\left(\partial-T r_{\min }+2(1-\alpha)\right)}{\left(\partial+(1-2 \alpha) T r_{\max }\right)\left(\partial-T r_{\min }+n(1-\alpha)\right)}
$$

Equality occurs for both the inequalities if and only if $G$ is a transmission regular graph. 
Proof. By given $X=\left(x_{1}, x_{2}, \ldots, x_{n}\right)^{T}$ is the unit eigenvector corresponding to the eigenvalue $\partial(G)$ of the matrix $D_{\alpha}$, therefore from

$$
D_{\alpha} X=\partial X, \quad \sum_{k=1}^{n} x_{k}=1
$$

it follows that

$$
\begin{aligned}
& \partial x_{1}=\alpha \operatorname{Tr}_{1} x_{1}+(1-\alpha) \sum_{k=1, k \neq 1}^{n} d_{i k} x_{k} \\
& \Rightarrow\left(\partial-\alpha T r_{\max }\right) x_{1} \leq(1-\alpha) \sum_{k=1, k \neq 1}^{n} d_{i k} x_{2} \leq(1-\alpha) T r_{\max } x_{2} \\
& =(1-\alpha) T r_{\max }\left(1-x_{1}-x_{3}-\cdots-x_{n}\right) \\
& \leq(1-\alpha) \operatorname{Tr}_{\max }\left(1-x_{\max }-(n-2) x_{n}\right) .
\end{aligned}
$$

This shows that

$$
\left(\partial+(1-2 \alpha) T r_{\max }\right) x_{\max } \leq(1-\alpha) T r_{\max }\left(1-(n-2) x_{\min }\right) .
$$

Using the left hand inequality of (3.1) in (3.3), it follows that

$$
x_{\max } \leq \frac{(1-\alpha) T r_{\max }\left(\partial-\operatorname{Tr}_{\min }+2(1-\alpha)\right)}{\left(\partial+(1-2 \alpha) T r_{\max }\right)\left(\partial-T r_{\min }+n(1-\alpha)\right)} .
$$

This completes the proof of right hand inequality.

Again from the $i$-th equation of $D_{\alpha} X=\partial X$, we have

$$
\begin{gathered}
\partial(G) x_{i}=\alpha T r_{i} x_{i}+(1-\alpha) \sum_{k=1, k \neq i}^{n} d_{i k} x_{k} \\
\Rightarrow\left(\partial(G)-\alpha T r_{\max }\right) x_{i} \leq(1-\alpha) \sum_{k=1, k \neq i}^{n} d_{i k} x_{\max } .
\end{gathered}
$$

Summing over all $i$ and using the fact $\sum_{k=1}^{n} x_{k}=1$, we get

$$
\begin{gathered}
\left(\partial(G)-\alpha T r_{\max }\right) \leq(1-\alpha) \sum_{i=1}^{n} \sum_{k=1, k \neq i}^{n} d_{i k} x_{\max } \\
=(1-\alpha) 2 W x_{\max }
\end{gathered}
$$

From this the left hand inequality follows.

Equality case can be discussed similarly as in Theorem 3.1. That completes the proof. 
The following lower bound for maximal entry $x_{\max }$ and upper bound for the minimal entry $x_{\text {min }}$ of the unit Perron vector $X$ of the spectral radius $\rho$ of distance matrix $D(G)$ was obtained in [8]:

$$
x_{\max } \geq \frac{\rho+1-n}{2 W-n(n-1)} \quad \text { and } \quad x_{\min } \leq \frac{\rho+1-n}{2 W-n(n-1)},
$$

with equality if and only if $G$ is a transmission regular graph. We note that for the $(n-1)$ transmission regular graph $K_{n}$, each of these bounds become an indeterminate form. Therefore, for the complete graph $K_{n}$ these bounds and the bounds obtained by using these bounds in Section 3 of the paper [8] does not make any sense. However, if in the statement the complete graph is excluded, then these results always hold.

For $p>1$, let $X=\left(x_{1}, x_{2}, \ldots, x_{n}\right)^{T}$ be the $p$-norm normalized Perron vector corresponding to spectral radius $\partial(G)$ and let $Y=\left(y_{1}, y_{2}, \ldots, y_{n}\right)^{T}$ be the unit Perron vector corresponding to spectral radius $\partial(G)$ of the matrix $D_{\alpha}(G)$. It is easy to see that

$$
x_{i}=\frac{y_{i}}{\left(\sum_{i=1}^{n} y_{i}^{p}\right)^{\frac{1}{p}}}, \quad \text { for all } i=1,2, \ldots, n .
$$

From this it is clear that

$$
x_{\max }=\frac{y_{\max }}{\left(\sum_{i=1}^{n} y_{i}^{p}\right)^{\frac{1}{p}}} \quad \text { and } \quad x_{\min }=\frac{y_{\min }}{\left(\sum_{i=1}^{n} y_{i}^{p}\right)^{\frac{1}{p}}} .
$$

Using the fact

$$
\left(\sum_{i=1}^{n} y_{i}^{p}\right)^{\frac{1}{p}} \leq n^{\frac{1}{p}} y_{\max } \quad \text { and } \quad\left(\sum_{i=1}^{n} y_{i}^{p}\right)^{\frac{1}{p}} \geq n^{\frac{1}{p}} y_{\min }
$$

with equality if and only if $G$ is a transmission regular graph. This fact together with (3.5), it follows that from any bound for $y_{\max }$ and $y_{\min }$, we can obtain a bound for $x_{\max }$ and $x_{\min }$. Thus, corresponding to the bounds obtained in Theorems 3.1 and 3.2, we can obtain the bounds for the maximal and minimal entry in the $p$-norm normalized Perron vector $(p>1)$ corresponding to spectral radius $\partial(G)$.

A complete split graph, denoted by $C S_{t, n-t}$, is the graph consisting of a clique on $n-t$ vertices and an independent set on the remaining $t$ vertices, such that each vertex of the clique is adjacent to every vertex of the independent set. It is clear that the graph $C S_{t, n-t}$ is the join of complete graph $K_{n-t}$ and the empty graph $\overline{K_{t}}$, that is $C S_{t, n-t}=K_{n-t} \vee \overline{K_{t}}$.

The following gives an upper bound on the minimal entry of the Perron vector of the generalized distance spectral radius of a graph $G$. 
Theorem 3.3. Let $G$ be a connected graph of order $n$ and let $X=\left(x_{1}, x_{2}, \ldots, x_{n}\right)^{T}$ be the p-norm normalized Perron vector corresponding to spectral radius $\partial(G)$ of $D_{\alpha}(G)$ with $x_{1} \geq x_{2} \geq \ldots \geq$ $x_{n}$. If $p \geq 1$ and $\alpha \in[0,1)$, then

$$
\begin{aligned}
x_{\min }= & x_{n} \\
\leq & \min \left\{\left(\frac{(\partial-\alpha(\beta+n-2)-2(1-\alpha)(\beta-1))^{p}}{(n-\beta)(\partial-\alpha(\beta+n-2)-2(1-\alpha)(\beta-1))^{p}+\beta(1-\alpha)^{p}(n-\beta)^{p}}\right)^{\frac{1}{p}},\right. \\
& \left.\left(\frac{(\partial-\alpha(2 n+\beta)-n+\beta+1)^{p}}{\beta(\partial-\alpha(2 n+\beta)-n+\beta+1)^{p}+(n-\beta) \beta^{p}(1-\alpha)^{p}}\right)^{\frac{1}{p}}\right\},
\end{aligned}
$$

where $\beta$ is the independence number of $G$. Moreover, the equality holds in (3.6) if and only if $G \cong$ $C S_{\beta, n-\beta}$.

Proof. Since $G$ is a connected graph of order $n$ with independence number $\beta$, we can assume that $V(G)=A \cup B$, such that $A=\left\{v_{1}, v_{2}, \ldots, v_{\beta}\right\}$ and $B=\left\{v_{\beta+1}, v_{\beta+2}, \ldots, v_{n}\right\}$ and there are no two adjacent vertices in the set $A$. We can assume that $x_{i}=\min _{v_{k} \in A} x_{k}$, and $x_{j}=\min _{v_{k} \in B} x_{k}$. Since $D_{\alpha} X=\partial X$, we have

$$
\begin{aligned}
\partial x_{i} & =\alpha \operatorname{Tr}_{i} x_{i}+(1-\alpha) \sum_{k=1, k \neq i}^{\beta} d_{i k} x_{k}+(1-\alpha) \sum_{k=\beta+1}^{n} d_{i k} x_{k} \\
& \geq \alpha \operatorname{Tr}_{i} x_{i}+(1-\alpha) \sum_{k=1, k \neq i}^{\beta} d_{i k} x_{i}+(1-\alpha) \sum_{k=\beta+1}^{n} d_{i k} x_{j} \\
& \geq \alpha(2(\beta-1)+n-\beta) x_{i}+2(1-\alpha)(\beta-1) x_{i}+(1-\alpha)(n-\beta) x_{j},
\end{aligned}
$$

that is

$$
x_{i} \geq \frac{(1-\alpha)(n-\beta) x_{j}}{\partial-\alpha(\beta+n-2)-2(1-\alpha)(\beta-1)} .
$$

Similarly, we have

$$
\begin{aligned}
\partial x_{j} & \geq \alpha T r_{j} x_{j}+(1-\alpha) \sum_{k=1}^{\beta} d_{j k} x_{i}+(1-\alpha) \sum_{k=\beta+1, k \neq j}^{n} d_{j k} x_{j} \\
& \geq \alpha(n-1) x_{j}+\beta(1-\alpha) x_{i}+(1-\alpha)(n-\beta-1) x_{j},
\end{aligned}
$$

that is

$$
x_{j} \geq \frac{\beta(1-\alpha) x_{i}}{\partial-\alpha(2 n+\beta)-n+\beta+1} .
$$

It follows from normalization that

$$
\beta x_{i}^{p}+(n-\beta) x_{j}^{p} \leq 1 .
$$


Substituting (3.7) in (3.9), we obtain

$$
x_{j} \leq\left(\frac{(\partial-\alpha(\beta+n-2)-2(1-\alpha)(\beta-1))^{p}}{(n-\beta)(\partial-\alpha(\beta+n-2)-2(1-\alpha)(\beta-1))^{p}+\beta(1-\alpha)^{p}(n-\beta)^{p}}\right)^{\frac{1}{p}}
$$

and Substituting (3.8) in (3.9), we obtain

$$
x_{i} \leq\left(\frac{(\partial-\alpha(2 n+\beta)-n+\beta+1)^{p}}{\beta(\partial-\alpha(2 n+\beta)-n+\beta+1)^{p}+(n-\beta) \beta^{p}(1-\alpha)^{p}}\right)^{\frac{1}{p}} .
$$

Thus, we complete the first part of the proof.

Suppose that equality holds in (3.6). Then all inequalities in the above argument must be equalities. From equality in (3.7), we get

$$
\begin{aligned}
& x_{k}=x_{j}, \quad d_{i k}=1, \quad \text { for all } \quad v_{k} \in B \\
& \text { and } x_{k}=x_{i}, \quad d_{i k}=2, \quad \text { for all } \quad v_{k} \in A .
\end{aligned}
$$

From equality in (3.8), we get

$$
\begin{aligned}
& x_{k}=x_{j}, \quad d_{j k}=1, \quad \text { for all } \quad v_{k} \in B \\
& \text { and } \quad x_{k}=x_{i}, \quad d_{j k}=1, \quad \text { for all } \quad v_{k} \in A .
\end{aligned}
$$

Thus each vertex in $A$ is adjacent to all the vertices of the set $B$ and each vertex in $B$ is adjacent to all the remaining vertices in $B$. Hence we must have $G \cong C S_{\beta, n-\beta}$.

Conversely, if $G \cong C S_{\beta, n-\beta}$, then it can be easily seen that equality occurs in (3.6). That completes the proof.

The following observation can be found in [23].

Lemma 3.1. [23] If $a_{1}, a_{2}, \ldots, a_{n}$ are positive numbers, then,

$$
\frac{b_{1}+b_{2}+\cdots+b_{n}}{a_{1}+a_{2}+\cdots+a_{n}} \leq \max _{i} \frac{b_{i}}{a_{i}}
$$

for any real numbers $b_{1}, b_{2}, \ldots, b_{n}$. Equality holds if and only if all the ratios $\frac{b_{i}}{a_{i}}$ are equal.

The following gives an upper bound on the maximal entry in the Perron vector of the generalized distance matrix in terms of minimum transmission degree, maximum transmission degree, diameter and spectral radius $\partial(G)$ of $D_{\alpha}(G)$. 
Theorem 3.4. Let $G$ be a connected graph of order $n$ having diameter d and let $X=\left(x_{1}, x_{2}, \ldots, x_{n}\right)^{T}$ be the p-norm normalized Perron vector corresponding to spectral radius $\partial(G)$ of $D_{\alpha}(G)$ with $x_{1} \geq x_{2} \geq \ldots \geq x_{n}$. Let $\alpha \in[0,1)$. If $p=1$, then

$$
x_{\max }=x_{1} \leq \frac{d(1-\alpha)}{\partial-\alpha T r_{\max }+d(1-\alpha)},
$$

and if $p>1$, then

$$
x_{\max }=x_{1} \leq\left(\frac{d(1-\alpha)\left(\partial-\alpha T r_{\min }\right)^{p-1}}{(d(1-\alpha))^{p-1}\left(\partial-\alpha T r_{\max }\right)+d(1-\alpha)\left(\partial-\alpha T r_{\min }\right)^{p-1}}\right)^{\frac{1}{p}} .
$$

For $p=1$, equality occurs if and only if $G \cong K_{n}$.

Proof. Since $D_{\alpha} X=\partial X$, for $v_{1} \in V(G)$, we have

$$
\begin{aligned}
& \partial x_{1}=\alpha \operatorname{Tr}_{1} x_{1}+(1-\alpha) \sum_{k=2}^{n} d_{1 k} x_{k} \\
& \Rightarrow\left(\partial-\alpha T r_{1}\right) x_{1}=(1-\alpha) \sum_{k=2}^{n} d_{1 k} x_{k} .
\end{aligned}
$$

It follows from normalization that $\sum_{k=2}^{n} x_{k}^{p}=1-x_{1}^{p}$, then from (3.10) and Lemma 3.1, we get

$$
\frac{\left(\partial-\alpha T r_{1}\right) x_{1}}{1-x_{1}^{p}}=\frac{(1-\alpha) \sum_{k=2}^{n} d_{1 k} x_{k}}{\sum_{k=2}^{n} x_{k}^{p}} \leq \max _{k \in\{2, \ldots, n\}} \frac{(1-\alpha) d_{1 k} x_{k}}{x_{k}^{p}} \leq \max _{k \in\{2, \ldots, n\}} \frac{(1-\alpha) d}{x_{k}^{p-1}} .
$$

Let $j \in\{2, \ldots, n\}$ be the index such that $\max _{k} \frac{(1-\alpha) d}{x_{k}^{p-1}}=\frac{(1-\alpha) d}{x_{j}^{p-1}}$, then

$$
\frac{\left(\partial-\alpha T r_{\max }\right) x_{1}}{1-x_{1}^{p}} \leq \frac{\left(\partial-\alpha T r_{1}\right) x_{1}}{1-x_{1}^{p}} \leq \frac{d(1-\alpha)}{x_{j}^{p-1}}
$$

For $p=1$ this leads to

$$
x_{1} \leq \frac{d(1-\alpha)}{\partial-\alpha T r_{\max }+d(1-\alpha)} .
$$

If $p>1$, we have

$$
x_{j} \leq\left(\frac{d(1-\alpha)\left(1-x_{1}^{p}\right)}{x_{1}\left(\partial-\alpha T r_{\max }\right)}\right)^{\frac{1}{p-1}} .
$$

For the $j$-th row of $D_{\alpha}(G)$, we have

$$
\partial x_{j}=\alpha \operatorname{Tr}_{j} x_{j}+(1-\alpha) \sum_{k=1, k \neq j}^{n} d_{j k} x_{k},
$$


this gives that

$$
\left(\partial-\alpha T r_{\min }\right) x_{j} \geq\left(\partial-\alpha T r_{j}\right) x_{j} \geq d(1-\alpha) x_{1}
$$

From (3.11) and (3.12), we get

$$
\begin{aligned}
& x_{1} \leq \frac{\left(\partial-\alpha T r_{\min }\right)}{d(1-\alpha)} \cdot\left(\frac{d(1-\alpha)\left(1-x_{1}^{p}\right)}{x_{1}\left(\partial-\alpha T r_{\max }\right)}\right)^{\frac{1}{p-1}} \\
& \Rightarrow x_{1} \leq\left(\frac{d(1-\alpha)\left(\partial-\alpha T r_{\min }\right)^{p-1}}{(d(1-\alpha))^{p-1}\left(\partial-\alpha T r_{\max }\right)+d(1-\alpha)\left(\partial-\alpha T r_{\min }\right)^{p-1}}\right)^{\frac{1}{p}} .
\end{aligned}
$$

For $p=1$, it follows from Lemma 3.2 that equality occurs if and only if $d_{1 k}=d$ for all $k=$ $2,3, \ldots, n$ and $T r_{\max }=T r_{1}$. Since $d_{1 k}=d$, for all $k=2,3, \ldots, n$, it implies that $T r_{\max }=$ $T r_{1}=d(n-1)$. If $d=1$, then it is clear that $G \cong K_{n}$. So, suppose that $d \geq 2$, then $d_{1 k}=d$ for all $k=2,3, \ldots, n$ gives that distance from $v_{1}$ to all other vertices is at least two. Which implies that $v_{1}$ is an isolated vertex in $G$, which is not possible as $G$ is a connected graph. This completes the proof.

The following gives a lower bound for the maximal entry of the Perron vector corresponding to the spectral radius $\partial(G)$ in terms of minimum transmission degree, maximum transmission degree and spectral radius $\partial(G)$ of $D_{\alpha}(G)$.

Theorem 3.5. Let $G$ be a connected graph of order $n$ and let $X=\left(x_{1}, x_{2}, \ldots, x_{n}\right)^{T}$ be the $p$-norm normalized Perron vector corresponding to spectral radius $\partial(G)$ of $D_{\alpha}(G)$ with $x_{1} \geq x_{2} \geq \ldots \geq$ $x_{n}$. If $p \geq 1$ and $\alpha \in[0,1)$, then

$$
x_{\max }=x_{1} \geq\left(\frac{(1-\alpha) \partial^{p-1}}{(1-\alpha) \partial^{p-1}+\left(\partial-\alpha T r_{\min }\right) T r_{\max }^{p-1}}\right)^{\frac{1}{p}},
$$

with equality if and only if $G \cong K_{n}$.

Proof. Since $D_{\alpha} X=\partial X$, for $v_{1} \in V(G)$, we have

$$
\partial x_{1}=\alpha \operatorname{Tr}_{1} x_{1}+(1-\alpha) \sum_{k=2}^{n} d_{1 k} x_{k} \geq \alpha \operatorname{Tr}_{\min } x_{1}+(1-\alpha) \sum_{k=2}^{n} x_{k} .
$$

Now, we multiply both sides of (3.14) by $x_{2}^{p-1}$, and get

$$
\begin{gathered}
\partial x_{1} x_{2}^{p-1} \geq \alpha T r_{\min } x_{1} x_{2}^{p-1}+(1-\alpha) \sum_{k=2}^{n} x_{k} x_{2}^{p-1} \\
\geq \alpha T r_{\min } x_{1} x_{2}^{p-1}+(1-\alpha) \sum_{k=2}^{n} x_{k}^{p} \\
\Rightarrow \partial x_{1} x_{2}^{p-1} \geq \alpha T r_{\min } x_{1} x_{2}^{p-1}+(1-\alpha)\left(1-x_{1}^{p}\right)
\end{gathered}
$$




$$
\Rightarrow \quad x_{2} \geq\left(\frac{(1-\alpha)\left(1-x_{1}^{p}\right)}{x_{1}\left(\partial-\alpha T r_{\min }\right)}\right)^{\frac{1}{p-1}} .
$$

Also, for $v_{2} \in V(G)$, we have

$$
\partial x_{2}=\alpha \operatorname{Tr}_{2} x_{2}+(1-\alpha) \sum_{k=1, k \neq 2}^{n} d_{2 k} x_{k} \leq \alpha \operatorname{Tr}_{\max } x_{1}+(1-\alpha) \operatorname{Tr}_{\max } x_{1} \leq \operatorname{Tr}_{\max } x_{1}
$$

From (3.15) and (3.16), we get

$$
\begin{aligned}
& \partial\left(\frac{(1-\alpha)\left(1-x_{1}^{p}\right)}{x_{1}\left(\partial-\alpha T r_{\min }\right)}\right)^{\frac{1}{p-1}} \leq \operatorname{Tr}_{\max } x_{1} \\
\Rightarrow & x_{1}^{p} \geq \frac{(1-\alpha) \partial^{p-1}}{(1-\alpha) \partial^{p-1}+\left(\partial-\alpha T r_{\min }\right) T r_{\max }^{p-1}},
\end{aligned}
$$

which gives the lower bound in (3.13). Suppose that equality holds in (3.13). Then all inequalities in the above must be equalities. From equality in (3.15), we get $d_{1 k}=1, T r_{1}=T r_{\min }$ and $x_{2}=x_{k}$, for all $k=2,3, \ldots, n$. Also, from equality in (3.16), we get $T r_{2}=T r_{\max }$ and $x_{1}=$ $x_{2}=\cdots=x_{n}$. This shows that all the row sums of $D_{\alpha}(G)$ are equal, that is $\operatorname{Tr}_{1}=\operatorname{Tr}_{2}=\cdots=$ $T r_{n}$, and so $\partial=T r_{1}=n-1$. This shows that equality occurs in (3.13) if and only if $G$ is a transmission regular graph with $\operatorname{Tr}_{i}=n-1$, which is only possible if and only if $G \cong K_{n}$. This completes the proof.

Next, we obtain a lower bound for $x_{\max }$, in terms of minimum degree, diameter and spectral radius $\partial(G)$ of $D_{\alpha}(G)$.

Theorem 3.6. Let $G$ be a connected graph of order $n$ having diameter $d$. Fix $p \geq 1$, and let $X=\left(x_{1}, x_{2}, \ldots, x_{n}\right)^{T}$ be the $p$-norm normalized Perron vector corresponding to the spectral radius $\partial(G)$ of $D_{\alpha}(G)$ with $x_{1} \geq x_{2} \geq \ldots \geq x_{n}$. Let $\delta$ be the minimum degree of the graph $G$ and let $\alpha \in[0,1)$. Then

$$
x_{1}=x_{\max } \geq\left(\frac{\partial^{p-1}(1-\alpha)}{\left(\partial-\alpha T r_{\min }\right)((n-1) d-(d-1) \delta)^{p-1}+\partial^{p-1}(1-\alpha)}\right)^{\frac{1}{p}} .
$$

Moreover, the equality holds if and only if $G \cong K_{n}$.

Proof. Proof follows from Theorem 3.5 by using the fact

$$
T r_{\max } \leq \delta+(n-1-\delta) d
$$

Acknowledgements: We sincerely thank an anonymous referee whose valuable comments and suggestions resulted in the improvement to the presentation of this paper. The research of A. Alhevaz was in part supported by a grant from Shahrood University of Technology, and the research of G-X. Tian was in part supported by the National Natural Science Foundation of China (No. 11801521). 


\section{References}

[1] M. Aouchiche and P. Hansen, Distance spectra of graphs: a survey, Linear Algebra Appl., 458 (2014) 301-386.

[2] M. Aouchiche and P. Hansen, Two Laplacians for the distance matrix of a graph, Linear Algebra Appl., 439 (2013) 21-33.

[3] A. Alhevaz, M. Baghipur and E. Hashemi, Further results on the distance signless Laplacian spectrum of graphs, Asian-European J. Math., 11(5) (2018), Article ID: 1850066 (15 pages).

[4] A. Alhevaz, M. Baghipur, Hilal A. Ganie and S. Pirzada, Brouwer type conjecture for the eigenvalues of distance signless Laplacian matrix of a graph, Linear and Multilinear Algebra; https://doi.org/10.1080/03081087.2019.1679074

[5] A. Alhevaz, M. Baghipur, Hilal A. Ganie and Y. Shang, Bounds for the generalized distance eigenvalues of a graph, Symmetry, 11(1529) (2019);doi:10.3390/sym11121529.

[6] A. Alhevaz, M. Baghipur, Hilal A. Ganie and Y. Shang, On the generalized distance energy of graphs, Mathematics, 8(17) (2020); doi:10.3390/math8010017.

[7] A. Alhevaz, M. Baghipur, Hilal A. Ganie and Y. Shang, The generalized distance spectrum of the join of graphs, Symmetry, 12(169) (2020); doi:10.3390/sym12010169.

[8] F. Atik and P. Panigrahi, Bounds on maximal and minimal entries of the $p$-normalized principal eigenvector of the distance and distance signless Laplacian matrices of graphs, Graphs and Combinatorics, https://doi.org/10.1007/s00373-018-1927-3

[9] S. Y. Cui, J. X. He and G. X. Tian, The generalized distance matrix, Linear Algebra Appl., 563 (2019), 1-23.

[10] S. M. Cioabă, A necessary and sufficient eigenvector condition for a graph to be bipartite, Electron. J. Linear Algebra, 20 (2010), 351-353.

[11] S. M. Cioabă, and D. A. Gregory, Principal eigenvectors of irregular graphs, Electron. J. Linear Algebra, 16 (2007), 366-379.

[12] K. C. Das, A sharp upper bound on the maximal entry in the principal eigenvector of symmetric nonnegative matrix, Linear Algebra Appl., 431 (2009), 1340-1350.

[13] K.C. Das, Maximal and minimal entry in the principal eigenvector for the distance matrix of a graph, Discrete Math., 311 (2011), 2593-2600. 
[14] K.C. Das, C.M.S. Junior, M.A. Freitas, R.R. Del-Vecchio, Bounds on the entries of the principal eigenvector of the distance signless Laplacian matrix, Linear Algebra Appl., 483 (2015), 200-220.

[15] X. Duan and B. Zhou, Sharp bounds on the spectral radius of a nonnegative matrix, Linear Algebra Appl., 439(10) (2013), 2961-2970.

[16] S. Pirzada, Hilal A. Ganie, A. Alhevaz and M. Baghipur, On spectral spread of generalized distance matrix of a graph, Linear and Multilinear Algebra, https://doi.org/10.1080/03081087.2020.1814194

[17] R. Li, The largest eigenvalue and some Hamiltonian properties of graphs, Electr. J. Linear Algebra, 34 (2018), 389-392.

[18] X. Li, Y. Fan and S. Zha, A lower bound for the distance signless Laplacian spectral radius of graphs in terms of chromatic number, J. Math. Research Appl., 34 (2014), 289-294.

[19] H. Lin and B. Zhou, The distance spectral radius of trees, Linear Multilinear Algebra, 67(2) (2019), 370-390.

[20] S. Liu and J. Shu, On the largest distance (signless Laplacian) eigenvalue of nontransmission-regular graphs, Electr. J. Linear Algebra, 34 (2018), 459-471.

[21] L. Lu, Q. Huang and X. Huang, On graphs whose smallest distance (signless Laplacian) eigenvalue has large multiplicity, Linear Multilinear Algebra, 66(11) (2018), 2218-2231.

[22] B. Ning and X. Peng, The Randić index and signless Laplacian spectral radius of graphs, Discrete Math., 342 (2019), 643-653.

[23] B. Papendieck and P. Recht, On maximal entries in the principal eigenvector of graphs, Linear Algebra Appl., 310 (2000), 129-138.

[24] S. Wasserman and K. Faust, Social Network Analysis, in: Structural Analysis in the Social Sciences, vol. 8, Cambridge University Press, Cambridge, 1997.

[25] Y. Wang and B. Zhou, On distance spectral radius of hypergraphs, Linear Multilinear Algebra, 66(11) (2018), 2232-2246.

[26] R. Xing and B. Zhou, On the distance and distance signless Laplacian spectral radii of bicyclic graphs, Linear Algebra Appl., 439 (2013), 3955-3963.

[27] R. Xing, B. Zhou and J. Li, On the distance signless Laplacian spectral radius of graphs, Linear Multilinear Algebra, 62 (2014), 1377-1387. 
[28] S. Zhao and Y. Hong, On the bounds of maximal entries in the principal eigenvector of symmetric nonnegative matrix, Linear Algebra Appl., 340 (2002), 245-252.

Abdollah Alhevaz Faculty of Mathematical Sciences, Shahrood University of Technology, P.O. Box: 316-3619995161, Shahrood, Iran

E-mail: a.alhevaz@shahroodut.ac.ir

Maryam Baghipur Department of Mathematics, University of Hormozgan, Bandar Abbas, Iran

E-mail: maryamb8989@gmail.com

Hilal Ahmad Ganie Department of Mathematics, University of Kashmir, Srinagar, India E-mail: hilahmad1119kt@gmail.com

Gui-Xian Tian Department of Mathematics, Zhejiang Normal University, Jinhua, Zhejiang, 321004, P.R. China

E-mail: guixiantian@163.com 